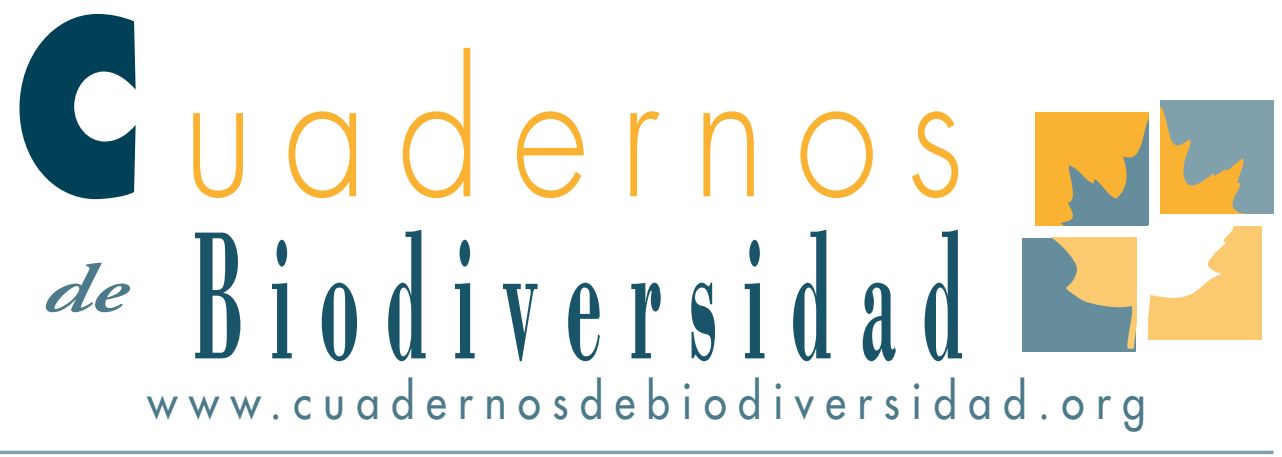

\title{
Colecciones ex situ de planta viva para la conservación de la planta amenazada Silene hifacensis, Rouy ex Willk (Caryophyllaceae)
}

P. Ferrer-Gallego ${ }^{1,2}$, I. Ferrando ${ }^{1,2}$, F. Albert ${ }^{1,2}$, V. Martínez ${ }^{1,2,}$ M. C. Escribä ${ }^{1,2}$, A. J. Navarro ${ }^{1,2}$, M. Pereira ${ }^{3}$, L. Viciano ${ }^{3}$, R. Carchano $^{2,4}$, J. Pérez ${ }^{2,4}$, I. López-Astilleros ${ }^{2,5}$, A. M. Vives ${ }^{2,5}$, G. Ballester $^{6}$ \& E. Laguna ${ }^{1,6}$

i Servicio de Vida Silvestre. Generalitat Valenciana. CiEF (Centro para la Investigación y Experimentación Forestal), Generalitat Valenciana, Av. Comarques del País Valencià, i i 4, E-46930, Quart de Poblet, Valencia, España.

2 VAERSA, C/ Mariano Cuber, i7, E-460 i i, Valencia.

3 Parque Natural El Montgó, Generalitat Valenciana. Camí Sant Joan, i, E-03700, Dénia, Alicante, España.

4 Servicio de Vida Silvestre. Dirección Territorial de Medio Ambiente de Alicante. Generalitat Valenciana, C/ Gerona, 26, E-0300i, Alicante, España

5 Parque Natural Peñón de Ifac, Generalitat Valenciana. C/ Illa de Formentera s/N, E-037io, Calpe, Valencia.

6 Servicio de Vida Silvestre, Generalitat Valenciana. C/ Castán Tobeñas, 77, Ciudad Administrativa 9 de Octubre-Torre i, E-460 i 8, Valencia, España.

\section{ABSTRACT}

The establishment of seed orchards has allowed obtaining a great deal of germoplasm of Silene hifacensis, an endangered endemic Ibero-Balearic species. In four years, 3.958.531 seeds have been collected in our four seed orchards from a total of 570 plants/ year per average, including all genetic variability from natural populations of this species in Alicante province (Illot of Mona, the Pessebret, Cova de les Cendres, Morro de Toix). 


\section{KEY WORDS:}

Conservation ex situ, endemism, germination, Silene hifacensis, endangered species, seed orchards.

\section{INTRODUCCIÓN}

No son pocos los casos en los que las especies se presentan en un determinado territorio con unas pocas poblaciones que, además contienen un bajo número de individuos (Bañares \& al., 2004). En materia de conservación, este ejemplo se complica si es fruto de un proceso de declive poblacional que, además, la mayoría de las veces va asociado a una pérdida de capacidad reproductiva y efectividad en el reclutamiento de nuevos efectivos (Bañares, 2003; Aguilar \& al., 2008).

En esta complicada situación para la conservación, las estrategias a seguir suelen ser abordadas desde una perspectiva de conservación integral a través del modelo in situ / ex situ / in situ, que engloba tanto actividades pasivas, mediante la preservación del hábitat, como activas, en las que se cuenta con la producción de nuevos individuos para la mejora de las poblaciones naturales (Falk, 1987; Guerrant, 1992, 1996; Guerrant \& Pavlik, 1997; Laguna, 2002). Esta producción y posterior restitución debe de ir precedida de la recolección de germoplasma (semillas o unidades de propagación) y su posterior manejo y conservación (Schoen \& Brown, 2001; Linington \& Pritchard, 2001; Heywood \& Iriondo, 2003; Walters, 2004). En este segundo aspecto, concretamente, en el trabajo con especies vegetales, gracias a determinadas características intrínsecas de algunos órganos de las plantas, como la totipotencia celular y la latencia, las colecciones de germoplasma tienen un papel fundamental en este escenario de conservación (Hawkes, 1987; Sarasan \& al., 2006; Ferrer-Gallego \& al., 2013).

Entre estas colecciones, las de planta viva constituyen un banco de germoplasma de gran utilidad, pues permiten la obtención de material genético de reproducción de manera continuada a lo largo del tiempo (Gómez-Campo, 1985; Cohen \& al., 1991; Linington \& Pritchard, 2001; Maunder \& al., 2004a). Este modelo es de mayor interés cuando la especie objeto de trabajo muestra un bajo número de individuos, ya que permite que todos sus genotipos estén representados en la colección (Brown \& Briggs, 1991; Brown \& Marshall, 1995; von Bothmer \& Selberg, 1995). En este sentido, para tener éxito en el programa de mantenimiento y producción de material de reproducción a partir de colecciones ex situ, se requiere cumplir al menos tres requisitos fundamentales (Prance, 1997; Maunder \& al., 2001; IPGRI, 2004; Guerrant \& al., 2004): 1) que realmente las colecciones conserven y representen una copia de seguridad útil para efectuar actividades in situ; 2) que preserven de forma representativa la diversidad genética y 3 ) que multipliquen de manera efectiva material para hacer frente a las demandas del trabajo in situ.

La confección de cultivos-colección ex situ de plantas necesita en muchos casos una adaptación de los modelos teóricos a la realidad de cada especie, ya que el origen diverso y las características tan dispares que pueden mostrar las especies que necesitan de estos programas hace que cada vegetal requiera ser estudiado y abordado por separado, con programas específicos, desde la germinación hasta el cultivo en vivero, y diseñado y aplicado de manera individual para poder extraer el máximo partido al casi siempre escaso material de partida con el que comienzan a funcionar, sobre todo en el caso de especies amenazadas. El establecimiento de huertos productores de semilla o material vegetativo con planta madre a partir de este tipo de colecciones constituye un paso adicional de la viverización, para el que suele carecerse de bibliografía o antecedentes de referencia. El gestor de este tipo de colecciones de planta viva ha de tener en cuenta que las condiciones en las que se localizan los ejemplares de una especie amenazada en el medio natural a menudo no son necesariamente las más idóneas para su supervivencia, sino aquellas en las que han quedado sus últimos reductos, tras haber desaparecido en muchos casos de hábitats óptimos. Por ello, es probable que los requerimientos de las plantas adultas en campo no sean precisamente los más adecuados para emular ex situ, o que incluso 
las plantas cultivadas muestren comportamientos que sólo aparecen cuando las plantas tienen unas condiciones inhabituales en la naturaleza.

Es preciso remarcar, que esta técnica no está exenta de ciertos inconvenientes; debido a que necesita de amplios espacios para algunas especies -que en ocasiones pueden ser muy grandes en el caso de especies arbóreas o colecciones con gran número de plantas-, o por otro lado, la alta exposición que puede tener la colección a enfermedades y plagas, el envejecimiento de las muestras, la hibridación espontánea, el empobrecimiento genético, las características del terreno de implantación, la mano de obra y el coste elevado que comporta el mantenimiento de algunas de estas colecciones, entre otras (Hamilton, 1994; Hurka, 1994; Maunder \& al., 2004b).

Por otra parte, algo de gran importancia en los proyectos de conservación ex situ a través de colecciones vivas, es que las muestras deben someterse a un modelo de continua renovación, favoreciendo la constante mezcla entre los diferentes ejemplares que formen parte de la misma población y si es posible incluyendo periódicamente nuevas plantas generadas tras nuevas germinaciones de material recolectado en campo. Ello asegura que se pierda el menor número posible de alelos, y reduce los riesgos genéticos de retrocruzamiento. En algunos casos, además, se deben realizar por ello polinizaciones cruzadas artificiales.

Las colecciones de planta viva pueden tener finalidad de exhibición, como las que suelen existir en instalaciones ecoeducativas y jardines botánicos, sin embargo, el modelo puede ser harto complejo, como anteriormente se ha indicado, cuando se integra a formar parte de los objetivos más concretos y cuya finalidad última es la recuperación in situ de poblaciones amenazadas (Cugnac, 1953; Heywood, 1991). Los modelos más típicos son los denominados huertos de progenies, donde el material representativo de cada población original se cultiva en lo posible por separado. Cuando se trata de material que ya ha sido obtenido en campo por vía vegetativa, al no ser posible a partir de semilla o resultar ésta excesivamente compleja y costosa para rendir en último término resultados similares, se habla a menudo de bancos de clones o huertos clonales (Pardos \& Gil, 1986).
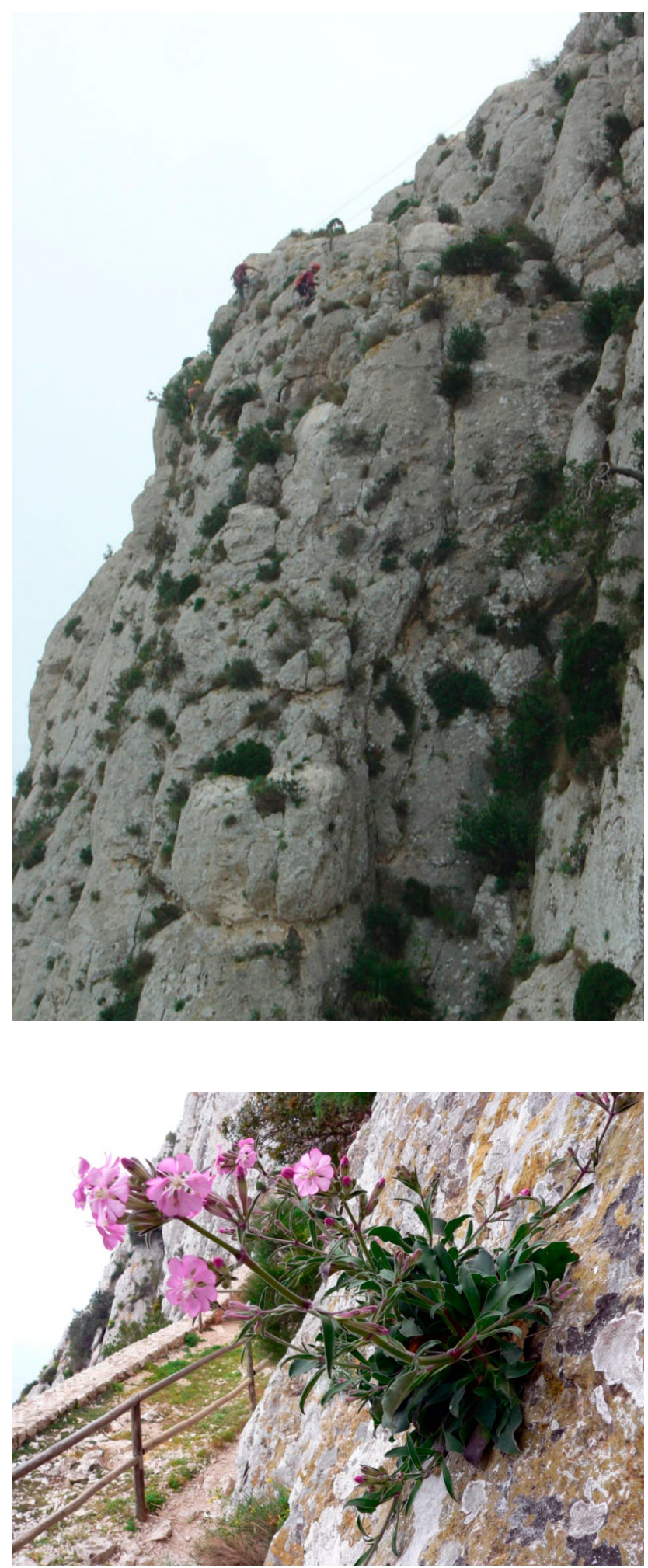

Trabajos in situ en la población de S. hifacensis del Penyal d'Ifac 
EL MODELO DE PRODUCCIÓN DE SEMILLAS EX SITU PARA SILENE HIFACENSIS

Silene hifacensis Rouy ex Willk. (silene d'Ifac) es una cariofilácea endémica íbero-balear, presente en los acantilados litorales del norte de Ibiza y cuadrante nororiental de Alicante (Cardona et al., 1987; Barber \& Sala, 1990; Banyuls et al., 1994). En el territorio valenciano la especie está catalogada "En peligro de extinción" existiendo solo cuatro núcleos poblacionales naturales actualmente (Aguilella et al., 1994, 2009; Anónimo, 2009; Blasco et al., 2011; Anónimo, 2013), todos con bajo número de efectivos que en suma no alcanzan la centena de individuos y separados en su mayoría por grandes distancias que impiden el flujo genético. Se trata de la única especie vegetal valenciana que dispone hasta el momento de un plan de recuperación legalmente aprobado (Anónimo, 2008). El citado plan contempla, entre otras acciones, el establecimiento de una colección de planta viva para el abastecimiento y la producción masiva de semillas, de las que una gran parte serán incorporadas al medio natural, bien mediante siembras directas, bien en forma de plántula (véase Sáinz-Ollero \& Hernández-Bermejo, 1979).

El principal objetivo de este modelo de producción es obtener una alta cantidad de semilla a partir de ejemplares-madre procedentes en un principio de semillas de las poblaciones naturales. Posteriormente las semillas se utilizan en los diferentes trabajos de conservación in situ y ex situ (Laguna et al., 2012). En el caso concreto de S. hifacensis, el modelo de huerto semillero es altamente recomendable, ya que: 1) es una especie que habita en lugares muy inaccesibles, como son los acantilados litorales, con el agravante que eso supone en las tareas de recolección, 2) poblaciones con un bajo número de efectivos, lo que puede llegar a suponer desequilibrios en la dinámica poblacional si existe

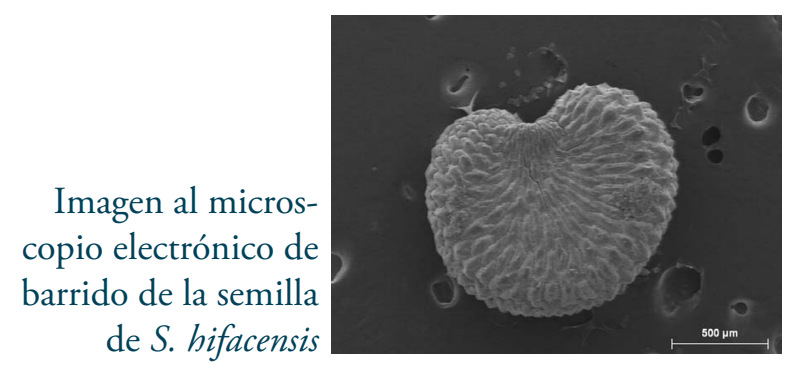

una recolección continuada de semillas todos los años, 3) es autocompatible y además no muestra depresión por endogamia acompañado de altas tasas de germinación (Nebot, 2012).
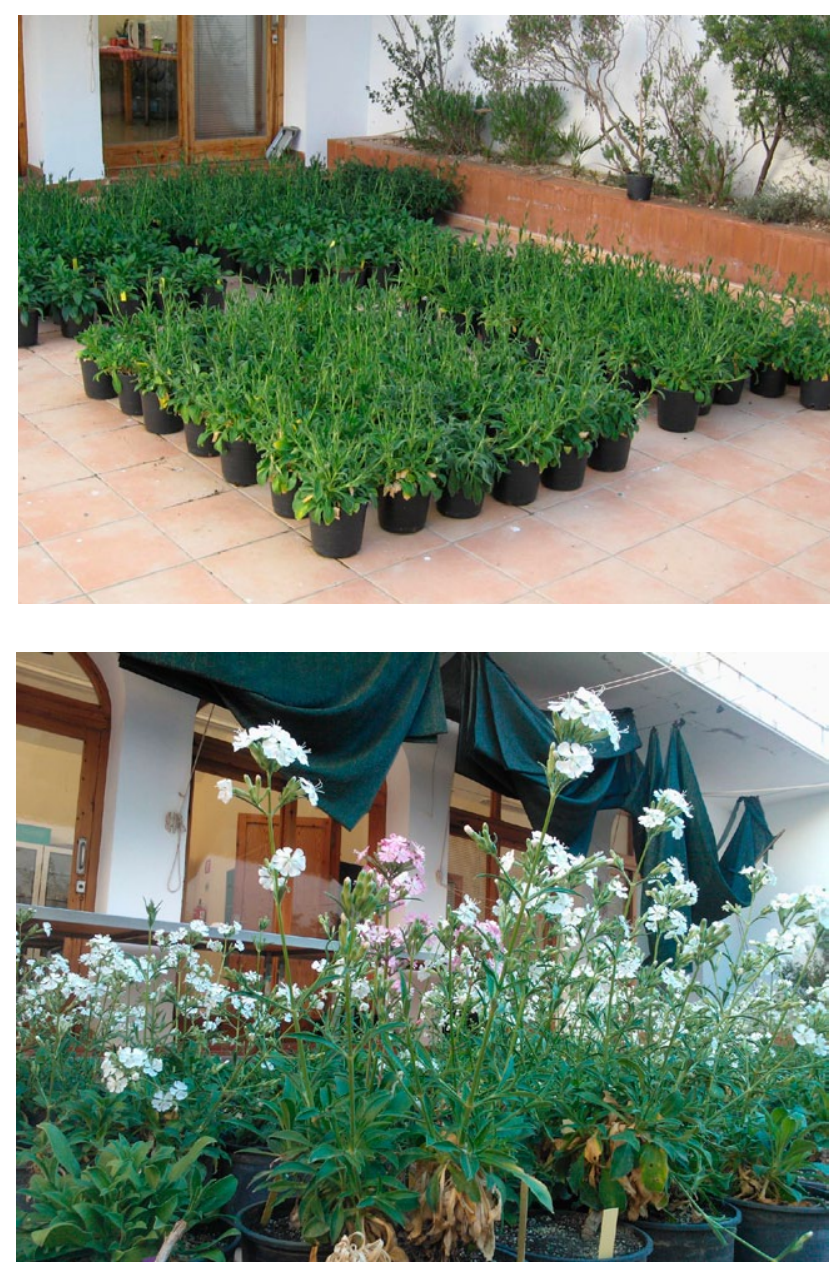

Colección de planta en uno de los huertos semilleros diseñados para la especie

Así pues, en 2009 se inició la puesta en marcha del programa de producción de semillas de esta especie mediante colecciones de huertos semillero (Ferrer-Gallego et al., 2013). En un primer término se recolectó material en todas las poblaciones naturales que, posteriormente se sustituyen por otros ejemplares que proceden tanto de las poblaciones silvestres (semillas recolectadas en otros años) como por individuos procedentes de semillas del propio huerto (generaciones filiares). Con este planteamiento se consigue una alta mezcla de material genético entre individuos y genotipos, incluso mayor del que podría ocurrir en el medio natural. 


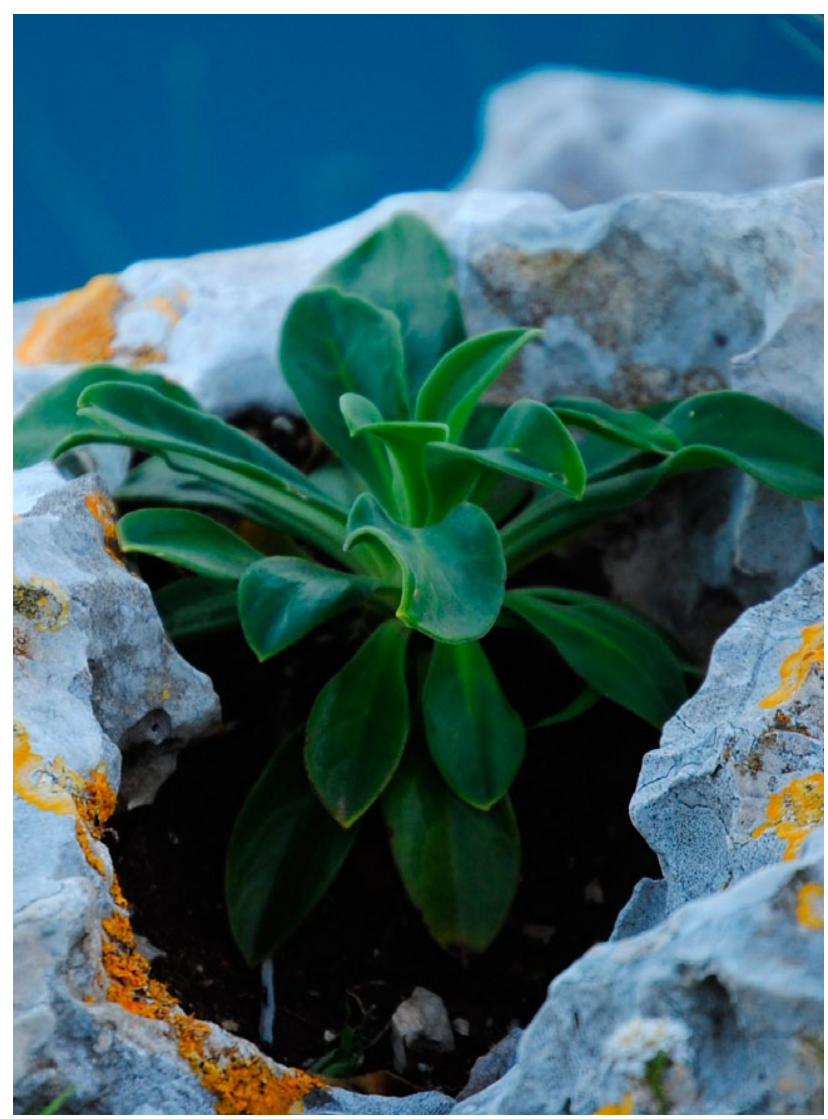

Ejemplar de S. hifacensis introducido en el medio natural y detalle de la flor

Las cuatro poblaciones valencianas objeto de trabajo; Illot de la Mona (Jávea), El Pessebret y Cova de les Cendres (Teulada-Moraira) y Morro de Toix (Calpe), se han repartido entre diferentes centros de la administración valenciana, que se encargan del mantenimiento y gestión de los diferentes huertos. Estos centros son: el Parque Natural El Montgó (Denia, Alicante), CIEF (Quart de Poblet, Valencia), el Parque Natural Penyal d'Ifac (Calpe, Alicante) y el vivero de planta forestal situado en la localidad de Santa Faç (Alicante), respectivamente. En cada uno de estos huertos el número inicial de plantas-madre fue de 50, pero este número se ha visto incrementado en la mayoría de los casos en los que se dispone de suficiente espacio e instalaciones, en parte debido al buen funcionamiento que están teniendo y la calidad del germoplasma que se obtiene (medido según capacidad germinativa, velocidad de germinación y calidad de planta viverizada -fitness) (Tabla 1).

\section{CANTIDAD Y CALIDAD DE LAS SEMI- LLAS PRODUCIDAS EN LOS HUERTOS SEMILLEROS}

Tras cuatro ańos de mantenimiento de los huertos (2009-2013) se ha alcanzado en suma 3.449,46 g de semillas y un total de 3.958.531 semillas. En la tabla 2 se detallan el peso y numero de las semillas obtenidas cada ańo).

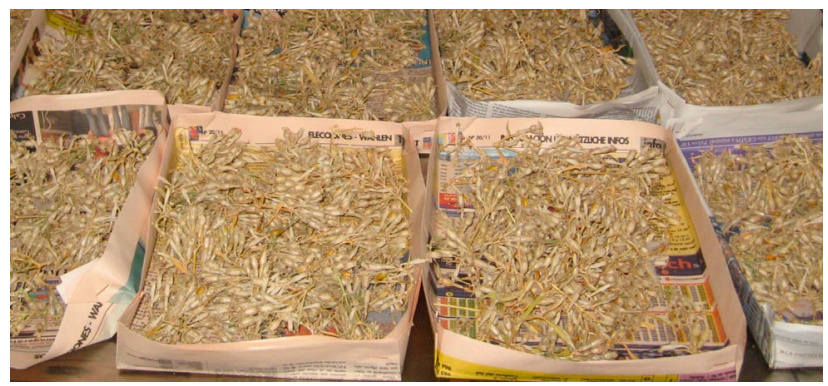

Cosecha de semillas obtenidas en 2012 a partir de los huertos semilleros

Tabla 1. Cantidad de planta-madre en cada uno de los cuatro huertos semilleros de Silene hifacensis durante el periodo $2010-2013$

\begin{tabular}{l|c|c|c|c|c} 
& \multicolumn{4}{|c|}{ Poblaciones } & \\
\hline No Plantas & Illot de la Mona & El Pessebret & Cova de les Cendres & Morro de Toix & Total \\
\hline 2010 & 50 & 50 & 50 & 50 & 200 \\
\hline 2011 & 50 & 200 & 176 & 60 & 486 \\
\hline 2012 & 50 & 250 & 420 & 190 & 910 \\
\hline 2013 & 50 & 175 & 304 & 156 & 685
\end{tabular}




\begin{tabular}{|c|c|c|c|c|c|c|}
\hline \multirow[b]{2}{*}{ Parámetros } & \multirow[b]{2}{*}{ Años } & \multicolumn{4}{|c|}{ Poblaciones } & \multirow[b]{2}{*}{ Total } \\
\hline & & $\begin{array}{c}\text { Illot de la } \\
\text { Mona }\end{array}$ & El Pessebret & $\begin{array}{l}\text { Cova de les } \\
\text { Cendres }\end{array}$ & Morro de Toix & \\
\hline \multirow{5}{*}{ P semillas $(\mathrm{g})$} & 2010 & 21,96 & 21,96 & 3,69 & 0,43 & 48,05 \\
\hline & 2011 & 66,37 & 499,71 & 636,44 & 64,73 & $1.267,28$ \\
\hline & 2012 & 41,25 & 205,60 & 218,03 & 399,83 & 864,71 \\
\hline & 2013 & 55,13 & 193,14 & 426,05 & 595,10 & $1.269,42$ \\
\hline & \multicolumn{5}{|c|}{ Total } & $3.449,46$ \\
\hline
\end{tabular}

\begin{tabular}{c|l|c|c|c|c|c}
\hline \multirow{4}{*}{ No semillas } & 2010 & 25.501 & 87.138 & 4.345 & 510 & 117.494 \\
\cline { 2 - 7 } & 2011 & 78.116 & 587.892 & 748.757 & 76.157 & 1.490 .922 \\
\cline { 2 - 7 } & 2012 & 61.351 & 172.788 & 218.759 & 430.590 & 883.488 \\
\cline { 2 - 7 } & 2013 & 78.897 & 174.002 & 608.966 & 604.762 & 1.466 .627 \\
\cline { 2 - 7 } & \multicolumn{5}{|c}{ Total } & 3.958 .531 \\
\hline
\end{tabular}

En lo que respecta a la calidad del germoplasma obtenido, los valores alcanzados mediante test de germinación realizados en placas de Petri en papel de filtro durante 30 días de ensayo en condiciones de $20 / 10^{\circ} \mathrm{C} 12 / 12$ horas luz/oscuridad, son en casi todos los casos superiores al $75 \%$, excepto tres recolecciones (Illot de la Mona en 2011 con un 61\%; Morro de Toix en 2012 con un 47\% y en 2011 con el 17\% de germinación) (Tabla 3). No obstante estos datos pueden ser atribuidos a la fecha de recolección del material y su falta de madurez, pues el análisis de viabilidad mediante el test de tetrazolio y la prueba de corte de semillas frescas reveló valores superiores al $80 \%$ de viabilidad para los tres lotes de semillas que expresan un relativo bajo porcentaje de germinación. Por otro lado, el peso de las semillas (peso de 100 unidades) varía entre los valores 0,061-0,105 gramos (Tabla 3). En lo referente a la pureza de las recolecciones, medida a partir de la razón entre semillas bien formadas y atrofiadas, los valores varían entre el 63,83\% y 98,12\% (Tabla 3). Los ensayos de germinación revelan que el tiempo en el que se alcanza el 50\% de las semillas germinadas (T50) es entre 3,4 y 9,8 días (Tabla 3). Por último, el fitness evaluado según supervivencia de plantas mantenidas durante 10 meses en cultivo bajo condiciones controladas de invernadero muestra porcentajes superiores al 85\% en todos los casos (Tabla 3). 


\begin{tabular}{|c|c|c|c|c|c|}
\hline \multirow{2}{*}{ Parámetros } & \multirow{2}{*}{ Años } & \multicolumn{4}{|c|}{ Poblaciones } \\
\hline & & Illot de la Mona & El Pessebret & Cova de les Cendres & Morro de Toix \\
\hline \multirow{4}{*}{ Pureza (\%) } & 2010 & 63,8 & 78,5 & 63,0 & 80,2 \\
\hline & 2011 & 77,6 & 71,4 & 94,9 & 90,7 \\
\hline & 2012 & 98,1 & 79,7 & 93,2 & 70,4 \\
\hline & 2013 & 95,2 & 80,2 & 94,5 & 95,4 \\
\hline \multirow{4}{*}{ P100 (g) } & 2010 & 0,06 & 0,09 & 0,06 & 0,10 \\
\hline & 2011 & 0,07 & 0,09 & 0,10 & 0,08 \\
\hline & 2012 & 0,07 & 0,09 & 0,09 & 0,06 \\
\hline & 2013 & 0,06 & 0,11 & 0,09 & 0,10 \\
\hline \multirow{4}{*}{$\% G$} & 2010 & $82 \pm 11,5$ & $98 \pm 2,3$ & $74 \pm 8,3$ & $91 \pm 5,0$ \\
\hline & 2011 & $61 \pm 3,9$ & $86 \pm 10,1$ & $95 \pm 1,9$ & $17 \pm 5,0$ \\
\hline & 2012 & $87 \pm 20,0$ & $77 \pm 7,8$ & $85 \pm 18,3$ & $47 \pm 7,6$ \\
\hline & 2013 & 100 & $92 \pm 6,5$ & $99 \pm 2,0$ & $99 \pm 2,0$ \\
\hline \multirow{4}{*}{$\mathrm{T} 50$} & 2010 & $9,6 \pm 1,1$ & $4,4 \pm 0,6$ & $3,4 \pm 0,6$ & $3,8 \pm 0,5$ \\
\hline & 2011 & $5,3 \pm 0,8$ & $5,1 \pm 0,6$ & $3,9 \pm 1,2$ & $9,8 \pm 0,9$ \\
\hline & 2012 & $4,4 \pm 0,3$ & $4,5 \pm 0,1$ & $4,2 \pm 0,9$ & $5,5 \pm 0,2$ \\
\hline & 2013 & $7,0 \pm 0,5$ & $4,6 \pm 0,4$ & $4,5 \pm 0,3$ & $6,7 \pm 0,1$ \\
\hline \multirow{4}{*}{ V no G } & 2010 & $4,2 \pm 3,2$ & $0,2 \pm 0,5$ & $5,5 \pm 1,3$ & $1,7 \pm 0,9$ \\
\hline & 2011 & $2,7 \pm 0,5$ & $3,5 \pm 2,5$ & $0,7 \pm 1,5$ & $12,0 \pm 2,0$ \\
\hline & 2012 & $1,5 \pm 1,0$ & $0,7 \pm 0,9$ & $0,2 \pm 0,5$ & $12,7 \pm 2,6$ \\
\hline & 2013 & 0 & $0,8 \pm 0,5$ & 0 & 0 \\
\hline \multirow{4}{*}{ Fitness (\%) } & 2010 & 85 & 90 & 88 & 92 \\
\hline & 2011 & 92 & 95 & 99 & 100 \\
\hline & 2012 & 90 & 97 & 95 & 96 \\
\hline & 2013 & 90 & 91 & 98 & 100 \\
\hline
\end{tabular}

Pureza: La pureza de la muestra consiste en conocer qué cantidad de semillas bien formadas en tanto por ciento hay dentro de una accesión, relación entre: semillas bien formadas/ semillas malformadas.

P100: Peso de 100 semillas.

$\%$ G: Porcentaje final de germinación después de 30 días de ensayo.

T50: Parámetro de velocidad del proceso de germinación; valor medido en días en el que se alcanza el 50\% del total de las semillas germinadas al final del ensayo (30 días).

V no G: Semillas viables que no han germinado. Estas se- millas suelen tener algún tipo de dormición o malformación embrionaria, por la que bajo condiciones ambientales adecuadas para la germinación, ésta no ocurre.

Fitness: Porcentaje de supervivencia de las plantas después 10 meses de cultivo tras la germinación en condiciones controladas de invernadero. Número total de plantas cultivadas por procedencia $=200$.

Condiciones de ensayo: Placas de Petri en cámaras germinadoras durante 30 días a una temperatura de $20 / 10^{\circ} \mathrm{C}$ y fotoperiodo de 12/12 horas luz/oscuridad. 


\section{CONCLUSIONES}

Los huertos productores de Silene hifacensis han generado un gran número de semillas durante los cuatro años que están funcionando. Lo que ha permitido realizar trabajos seriados de restitución in situ, como el refuerzo y la creación de nuevas poblaciones. Así, los trabajos de plantaciones y siembras previas que se habían realizado desde 1992 hasta 2003, en suma un total de 7 actuaciones, todas con un bajo éxito de supervivencia en los ejemplares implantados y fundamentalmente dirigidos a reforzar las poblaciones naturales, se han visto incrementadas en los últimos años hasta 46 actuaciones lo que ha permitido elevar el número de poblaciones hasta 12 y el área de distribución de la especie en el territorio valenciano en un $78 \%$ (de 132 a $235 \mathrm{~km} 2$ ) y el número de ejemplares en un $75 \%$, pasando de 99 (censados en 2008) a 173 censados en 2013. Al mismo tiempo, estas colecciones han permitido realizar el estudio de la biología reproductiva de la especie (Nebot, 2012), información sin duda de gran valor para el buen funcionamiento del plan de recuperación.

Este modelo de producción ex situ es muy recomendable para esta especie debido al bajo número de individuos silvestres que requiere, dada la dificultad que supone desarrollar trabajos en acantilados de litoral donde se encuentran las poblaciones de la planta. Además, debido a determinadas características de la biología reproductiva de la especie, autocompatible, baja depresión por endogamia, alta producción de semillas/individuo y alta tasa de germinación de las semillas, las colecciones de los huertos semillero permiten incluso realizar una mejora genética del germoplasma generado en condiciones ex situ frente al existente in situ, ya que los cruces contralados entre progenies e individuos puede llega a ser mucho mayor que en las poblaciones naturales.

\section{REFERENCIAS}

Aguilar, R., Quesada, M., Ashworth, L., Herrerias-Diego, Y. \& Lobo, J. 2008. Genetic consequences of hábitat fragmentation in plant populations: susceptible signals in plant traits and methodological approaches. Mol. Ecol. 17: 5177-5188.

Aguilella, A., Carretero, J.L., Crespo, M.B., Figuerola, R. \& Mateo, G. 1994. Flora vascular rara, endémica o amenazada de la Comunidad Valenciana. Conselleria de Medi Ambient, Generaliᄀtat Valenciana. Valencia. 274 pp.

Aguilella, A., Fos, S. \& Laguna, E. (eds.). 2009. Catálogo Valenciano de Especies de Flora Amenazadas. Colección Biodiversidad, 18. Conselleria de Medi Ambient, Aigua, Urbanisme i Habitatge, Generalitat Valenciana. Valencia. 358 pp.

Anónimo 2008. Decreto 40/2008, de 4 de abril, del Consell, por el que se aprueba el Plan de Recuperación de la Silene de Ifac en la Comunitat Valenciana. Diari Oficial de la Comunitat Valenciana 5739: 56114-56128.

Anónimo 2009. Decreto 70/2009, de 22 de mayo, del Consell, por el que se crea y regula el Catálogo Valenciano de Especies de Flora Amenazadas y se regulan medidas adicionales de conserva $\neg$ ción. Diari Oficial de la Comunitat Valenciana 6021: 20143-20162.

Anónimo 2013. Orden 6/2013, de 25 de marzo, de la Conselleria de Infraestructuras, Territorio y Medio Ambiente, por la que se modifican los listados valencianos de especies protegidas de flora y fauna. Diari Oficial de la Comunitat Valenciana 6996: 8682-8690.

Banyuls, B., Sala, J. \& Soler, J.X. 1994. Silene hifacensis, una de las numerosas especies litorales a punto de desaparecer. Quercus 1: 32-33.

Bañares, A. (ed.). 2003. Biología de la conservación de plantas amenazadas. Ministerio de Medio Ambiente. Madrid. 270 pp.

Bañares, A., Blanca, G., Güemes, J., Moreno, J.C. \& Ortiz, S. (eds.). 2004. Atlas y libro rojo de la flora vascular amenazada de España. Táxones propritarios. Ministerio de Medio Ambiente. Madrid. 1070 pp.

Barber, A. \& Sala, J. 1990. Situació actual de l'endemisme boᄀtànic "Silene hifacensis" Rouy ex Willk. Aguaits 5: 55-60. 
Blasco, M.P., Fabado, J., Carrió, E., Güemes, J.F., Torres, N., Ballester, G., Fos, S., Pérez-Botella, J. \& Güemes, J. 2011. Silene hifacensis Rouy ex Willk. En: Bañares, A., Blanca, G., Güemes, J., Moreno, J.C. \& Ortiz, S. (eds.): Atlas y Libro Rojo de la flora vascular amenazada de España. Adenda 2010. Ministerio de Medio Ambiente y Medio Rural y Marino. Madrid. pp. 92-93.

Brown, A.D.H. \& Briggs, J.D. 1991. Sampling strategies for genetic variation in ex situ collections of endangered plant species. In: Falk, D.A. \& Holsinger, K.E. (eds.): Genetics and conservation of rare plants. Oxford University Press. New York. pp. 99-122.

Brown, A.D.H. \& Marshall, D.R. 1995. A basic sampling strategy: theory and practice. In: Guarino, L. \& Ramantha Rao, V.R. (eds.): Collecting plant genetic diversity: technical guidelines. CAB International Wallington. U.K. pp. 75-111.

Cardona, M.A., Torres, N. \& Gómez Campo, C. 1987. Silene hifacensis. En: Gómez Campo, C. \& al: Libro rojo de especies vegetales amenazadas de España peninsular e Islas Baleares. ICONA. pp. 576-577

Cohen, J.I., Williams, J.T., Plucknett, D.L. \& Shands, H.L. 1991. Ex situ conservation of plant genetic resources: global development and environmental concerns. Science 253: 866-872.

Cugnac, A. 1953. Le role des jardines botaniques pour la conservation des especes menacees de disparition ou d'alteration. Ann. Biol. 29: 361-367.

Falk, D.A. 1987. Integrated conservation strategies for endangered plants. Nat. Areas J. 7: 118-123.

Ferrer-Gallego, P.P., Ferrando, I., Gago, C. \& Laguna, E. (eds.) 2013. Manual para la conservación de germoplasma y el culativo de la flora valenciana amenazada. Colección Manuales Técnicos Biodiversidad, 3. Conselleria d'Infraestructures, Territori i Medi Ambient. Generalitat Valenciana. Valencia. 252 pp.

Guerrant, E.O.J. 1992. Genetic and demographic considerations in the sampling and reintroduction of rare plants. In: Fiedler, P.L. \& Jain, S.K. (eds.): Conservation biology: the theory and practice of nature conservation, preservation and management. Chapman \& Hall. New York. pp. 321-344.

Guerrant, E.O.J. 1996. Designing populations: demographic, genetic and horticultural dimensions. In: Falk, D.A., Millar, C.I. \& Olwell, M. (eds.): Restoring diversity. Strategies for reintroduction of endangered plants. Island Press. Washington. pp. 171-207.
Guerrant, E.O.J. \& Fiedler, P.L. 2004. Accounting for sample decline during ex situ storage and reintroduction. In: Guerrant, E.O.J., Havens, K. \& Maunder, M. (eds.): Ex situ plant conservation: supporting species survival in the wild. Island Press. Washington. pp. 365-386.

Guerrant, E.O.J. \& Pavlik, B.M. 1997. Reintroduction of rare plants: genetics, demography and the role of ex situ conservation methods. In: Fiedler, P.L. \& Kareiva, P.M. (eds.): Conservation biology for the coming decade. Chapman \& Hall. New York. pp. 80-108.

Hamilton, M.B. 1994. Ex situ conservation of wild plant species: time to reassess the genetic assumptions and implications of seed banks. Conserv. Biol. 8: 39-49.

Hawkes, J.G. 1987. A strategy for seed banking in botanic gardens. In: Bramwell, D., Hamann, O., Heywood, V.H. \& Synge, H. (eds.): Botanic gardens and the world conservation strategy. Academic Press. London. pp. 131-149.

Heywood, V. 1991. Botanic gardens and the conservation of medicinal plants. In: Akerele, O., Heywood, V. \& Synge, H. (eds.): Conservation of medicinal plants. Cambridge University Press. Cambridge. pp. 213-228.

Heywood, V.H. \& Iriondo, J.M. 2003. Plant conservation: old problems, new perspectives. Biol. Conserv. 113: 321-335

IPGRI/FAO/FLD 2004. Forest genetic resources conservation and management. Vol. 3. Plantations and genebanks. Biodiversity International. Rome.

Laguna, E. 2002. Estrategia de conservación de la flora silvestre en la Comunidad Valenciana. Conserv. Vegetal 7: 12-13.

Laguna, E., Ballester, G., Ferrer-Gallego, P.P., Pereira, M., Vicioso, L., Albert, F.J., Escribá, M.C., Ferrando, I., Navarro, A.J. \& Pérez-Botella, J. 2011. First phase of the re-introduction of Silene hifacensis (Caryophyllaceae) in Cap de Sant Antoni, Valencian Community, Spain. In Soorae, P.S. (ed.): Global Re-Introduction Perspectives: 2011. More case studies around the globe: 244-248. IUCN Re-Introduction Specialist Group. Gland \& Abu-Dabi.

Linington, S.H. \& Pritchard, H.W. 2001. Gene banks. In: Levin S.A. (ed.): Encyclopedia of biodiversity. Academic Press. San Francisco. pp. 165-181.

Maunder, M., Higgens, S. \& Culham, A. 2001. The effectiveness of botanic garden collections in supporting plant conservation: a European case study. Biodivers. Conserv. 10: 383-401. 
Maunder, M., Havens, K., Guerrant, E.O.J. \& Falk, D.A. 2004a. Ex situ methods: a vital but underused set of conservation resources. In: Guerrant, E.O.J., Havens, K. \& Maunder, M. (eds.): Ex situ plant conservation: supporting species survival in the wild. Island Press. Washington. pp. 389-408.

Maunder, M., Hughes, C., Hawkins, J.A. \& Culham, A. 2004b. Hybridization in ex situ plant collections: conservation concerns, liabilities, and opportunities. In: Guerrant, E.O.J., Havens, K. \& Maunder, M. (eds.): Ex situ plant conservation: supporting species survival in the wild. Island Press. Washington. pp. 325-364.

Nebot, A. 2012. Biología reproductiva del endemismo amenazado Silene hifacensis Rouy ex Willk. (Caryophyllaceae). Universitat de València. Inéd.

Pardos, J.A. \& Gil, L. 1986. Los huertos semilleros: Estudios básicos para su establecimiento en España. ICONA. Monografías 44: 128 pp.

Prance, G.T. 1997. The conservation of botanical diversity. In: Maxted, N., Ford-Lloyd, B.V. \& Hawkes, J.G. (eds.): Plant genetic conservation. The in situ approach. Chapman \& Hall. London. pp. 3-14.
Sáinz-Ollero, H. \& Hernández-Bermejo, J.E. 1979. Experimental reintroductions of endangered plant species in their natural habitats in Spain. Biol. Conserv. 16: 195-206.

Sarasan, V., Cripps, R., Ramsay, M.M., Atherton, C., McMichen, M., Prendergast, G. \& Rowntree, J.K. 2006. Conservation in vitro of threatened plants-progress in the past decade. In Vitro Cell. Dev. Biol. 42: 206-214.

Schoen, D.J. \& Brown, A.D.H. 2001. The conservation of wild plant species in seed banks. Bioscience 51: 960-966.

von Bothmer, R. \& Selberg, O. 1995. Strategies for the collecting of wild species. In: Guarino, L. \& Ramantha Rao, V.R. (eds.): Collecting plant genetic diversity: technical guidelines. CAB International Wallington, U.K. pp. 93-111.

Walters, C. 2004. Principles for preserving germplasm in gene banks. In: Guerrant, E.O.J., Havens, K. \& Maunder, M. (eds.): Ex situ plant conservation: supporting species survival in the wild. Island Press. Washington. pp. 113-138. 